Archives de sciences sociales des religions

149 | janvier-mars 2010

Varia

\title{
Le pèlerinage vers Saint-Jacques-de-Compostelle
}

\section{Elena Zapponi}

\section{OpenEdition}

Journals

Édition électronique

URL : http://journals.openedition.org/assr/21855

DOI : 10.4000/assr.21855

ISSN : 1777-5825

Éditeur

Éditions de l'EHESS

\section{Édition imprimée}

Date de publication : 31 mars 2010

Pagination : 73-87

ISBN : 978-2-7132-2253-5

ISSN : 0335-5985

Référence électronique

Elena Zapponi, « Le pèlerinage vers Saint-Jacques-de-Compostelle», Archives de sciences sociales des religions [En ligne], 149 | janvier-mars 2010, mis en ligne le 05 octobre 2010, consulté le 02 mai 2019. URL : http://journals.openedition.org/assr/21855; DOI : 10.4000/assr.21855 


\section{Elena Zapponi}

\section{Le pèlerinage vers Saint-Jacques-de-Compostelle}

El Camino francés est une route pèlerine qui va de Roncevaux à SaintJacques-de-Compostelle, en Galice. Née au IX ${ }^{\mathrm{e}}$ siècle, sous l'effet de la dévotion pour Jacques le Majeur, évangélisateur de l'Espagne, celui que l'on appelle «le fils du tonnerre ", peregrino et matamoros, ce lieu renouvelle son attrait et devient un indicateur du retour aux pèlerinages, pratiques volontaires, modulables, émotionnelles qui marquent notre époque contemporaine.

Jusqu'en 1984, plus ou moins, el Camino francés n'a mobilisé que quelques centaines de pèlerins certifiés (Bertrand, Muller, 1999 : 39-63). Depuis, la courbe de fréquentation est uniformément ascendante : la participation a augmenté de façon exponentielle de 1990 à aujourd'hui pour atteindre plus de cent mille personnes actuellement avec des pointes de près de cent mille pour l'année sainte de 1993, de cent cinquante mille pour l'année sainte de 1999 et de cent quatrevingt mille pour l'année sainte de 2004. Pendant les années saintes ${ }^{1}$, la participation est visiblement plus élevée que celle des autres années ; toutefois, l'augmentation des présences par rapport au flux général demeure relativement constante. Le cadre général des données statistiques indique que la participation pèlerine de 1990 à 2007 est passée de 4918 présences à $114026^{2}$, c'est-à-dire qu'elle s'est multipliée par vingt approximativement.

Pendant les quinze dernières années, la population pèlerine de Compostelle s'est diversifiée en ce qui concerne les nationalités et les classes d'âges des marcheurs. Jusqu'aux années quatre-vingts, les pèlerins venaient majoritairement d'un public européen, Compostelle, comme Rome et Jérusalem, représentant un lieu majeur de la chrétienté européenne. Aujourd'hui, les marcheurs viennent également de mondes éloignés de l'Espagne comme le Brésil, les États-Unis, l'Australie, le Japon ou le Canada. En outre, si le public des premières années de la décennie quatre-vingt-dix présentait une composition sociale principalement

1. Les années saintes sont célébrées tous les sept ans, lorsque la fête de saint Jacques, le 25 juillet, tombe un dimanche.

2. Je fais référence aux données de la Oficina de Sociologia del Arzobispado de Santiago de Compostela qui les élabore à partir du registre de la Oficina de Acogida del Peregrino. Voir le site www.archicompostela.org 
"bourgeoise bohème " (étudiants et professions libérales), le Chemin tend aujourd'hui à se massifier et à devenir un phénomène qui attire des acteurs d'appartenances sociales très variées. Les façons de croire et de "pèleriner » des marcheurs en sont d'autant plus diversifiées.

Si l'on peut représenter l'acte qui distingue la tradition du pèlerinage chrétiencatholique de la façon suivante $: A \longrightarrow B$, où le terme $A$ représente le lieu du départ et $B$ le lieu sanctuaire vers lequel le croyant oriente ses attentes, cette formule n'est pas toujours validée par le code de sens mis en place par les façons de "pèleriner » contemporaines. La démarche actuelle des pèlerins de Compostelle ne correspond pas toujours à la dynamique de l'attente indiquée par le signe précédent ; les notions de "ville sainte ", " saint ", " sanctuaire ", semblent être recomposées "à la carte " par un public dont l'imaginaire religieux est de plus en plus fragmenté. La notion de pèlerinage orienté vers la cathédrale de Santiago garde son importance, revitalisée par l'action parallèle des organisateurs de $c e$ pèlerinage et des acteurs qui valident $c e$ but du voyage. Mais la pratique de l'aller même prend actuellement une importance croissante : souvent, c'est el Camino qui devient attirant, c'est-à-dire l'expérience de la route et la possibilité que le pèlerinage offre de se représenter, pendant ce temps de passage, comme un sujet social in itinere.

L'analyse suivante, fondée sur trois campagnes de terrain qualitatif, menées in situ par postures successives et décentrées ${ }^{3}$, se propose de saisir la mobilité de la scène pèlerine et la pluralisation des croyances qui la caractérisent. Pour rendre compte de ce paysage et pour pénétrer dans l'espace concret du pèlerinage, on retiendra un angle d'analyse très précis, voire détaillé : "les objets des pèlerins ", ce que les pèlerins portent chaque jour avec eux, dans leur sac. En route, les marcheurs sont placés face à la même règle du jeu - marcher - et ils se trouvent à partager des conditions semblables : porter sur leurs épaules un bagage réduit à l'essentiel et être identifiés par le document que constitue la credencial del peregrino ${ }^{4}$. Ce laissez-passer, qui lie progressivement dans le temps l'identité du passeur à l'espace de son passage, remplace durant le voyage le passeport ou le carnet d'identité. Muni de cette lettre de créance - encore vierge des traces des lieux - et du sac sur les épaules, on entreprend le chemin. Ces deux éléments

3. J'ai analysé le pèlerinage en essayant de tenir compte à la fois de la logique organisationnelle des institutions qui en gèrent l'aménagement, de la logique des pèlerins qui l'expérimentent et de la logique des Espagnols installés, qui habitent cet espace en permanence. Par conséquent, j'ai alterné une ethnographie on the road $(1998,1999,2002)$ c'est-à-dire parcourir à pied la route du Camino francés pour pouvoir enquêter auprès des acteurs en marche, avec une ethnographie sur place, assumant le rôle d'hospitaliera, bénévole dans des gîtes pour pèlerins sur la route $(1999,2002)$.

4. Lettre de créance que l'on acquiert auprès des associations jacquaires internationales ou auprès de la Real Colegiata de Ronceveaux. Au fur et à mesure de la marche, on consigne sur cette carte les dates et les cachets des étapes de la route, les épreuves du trajet parcouru, permettant, une fois arrivé à Saint-Jacques-de-Compostelle, d'obtenir le certificat du pèlerinage accompli. 
- le bagage minimal et la credencial - suffisent à donner une indication de la spécificité du contexte de Compostelle : on quitte un document d'identité pour en adopter temporairement un autre, on quitte sa garde-robe quotidienne pour assumer un nouvel habit, concret et symbolique, celui du pèlerin.

Le lien entre la signification des objets des pèlerins et les recompositions identitaires qu'ils véhiculent constitue une entrée privilégiée pour observer le monde extraordinaire du Camino de Santiago et la réinvention individuelle de la tradition du pèlerinage catholique. Dans un contexte où la règle de l'aller s'avère essentielle, la valeur symbolique des objets du quotidien pèlerin augmente. Dans la situation de bagage minimal à laquelle on se réfère, les objets, tels des signes identitaires, parlent des trajectoires de leurs propriétaires : puisque la règle de l'itinérance oblige à un omnia mea mecum porto, ce que l'on amène avec soi et, il faut le souligner, sur soi, devient plus facilement un signe d'état de pèlerin et une expression de la quête de soi expérimentée sur la route vers Saint-Jacquesde-Compostelle.

\section{Des objets pèlerins aux sujets croyants}

La règle forte de la scène de Compostelle - aller à pied - et la contrainte du poids du sac obligent à faire un choix avant le départ et à fixer une image de soi par une tenue vestimentaire et des accessoires qui accompagneront la marche. La préparation du sac implique souvent un certain recueillement : on pourrait la définir comme une opération symboliquement grave du fait qu'elle consiste d'ailleurs en un acte de pesage matériel. Nombreux sont les acteurs qui racontent avoir mis sur une balance leur bagage et avoir longtemps hésité à éliminer quelques grammes ; comme si, au moment du passage vers la scène inconnue de la marche, le monde des objets s'animait, devenant un garant de "la présence de soi au monde »(De Martino, 1999 : 151-152).

Wolfgang, Autrichien de la région de Salzburg, âgé de trente ans, séparé et père d'un enfant, actuellement assistant social dans une association où il est chargé d'enseigner le jardinage aux personnes handicapées, décrit ainsi son passé professionnel : "D'abord j'ai appris à faire le boulanger. Je ne voulais pas aller à l'armée et puis j'ai travaillé dans le social et j'ai fait une école. »Dans son cas, le départ semble se faire selon une logique d'improvisation, sur l'écho des récits d'un ami et de la lecture du livre de Paulo Coelho ${ }^{5}$. Sa position croyante, qui

5. Paulo Coelho, Le pèlerin de Compostelle (O Diario de um mago), Paris, Éditions Anne Carrière, 1987. Le best-seller de Coelho est un exemple de la tendance biographique-initiatique inspirée par le Chemin de Compostelle. Cet auteur insiste sur la configuration d'une búsqueda (quête) tout au long du chemin magique ainsi que sur une trajectoire du croire autonome par rapport au pèlerinage catholique. Le cœur de cette individualisation du croire réside dans l'expérimentation personnelle tout au long de la route et dans la recherche d'une voie à l'intérieur de soi-même. Le chemin est, ainsi, configuré comme un lieu de rencontre avec un catholicisme dé-dogmatisé et autonome. 
fait la distinction entre la formation religieuse reçue et la pratique actuelle, est exprimée de la façon suivante : "Maintenant je ne suis pas un catholique, je suis un chrétien... Je pense que l'Église catholique est un grand business. Je le vois dans ma ville, qui est une ville très catholique. " Le départ de Wolfgang semble se faire le cœur léger : "J'avais le temps et alors je marche sur le Chemin. Je marche seul depuis Saint-Jean-Pied-de-Port. Ma copine m'a amené au train, puis elle a dit : "La distance jusqu'à Paris n'est pas grande !” et elle m'a conduit à Paris en voiture. " Mais la vocation de la route émerge par la suite comme déterminée par une nécessité de quête : "Je suis seulement en train de parcourir la route... mais il y a quelque chose que je veux éloigner de moi en marchant... et j'espère que, quand je retournerai dans ma ville, ou ma terre, je vais avoir la réponse, j’aurai trouvé... »

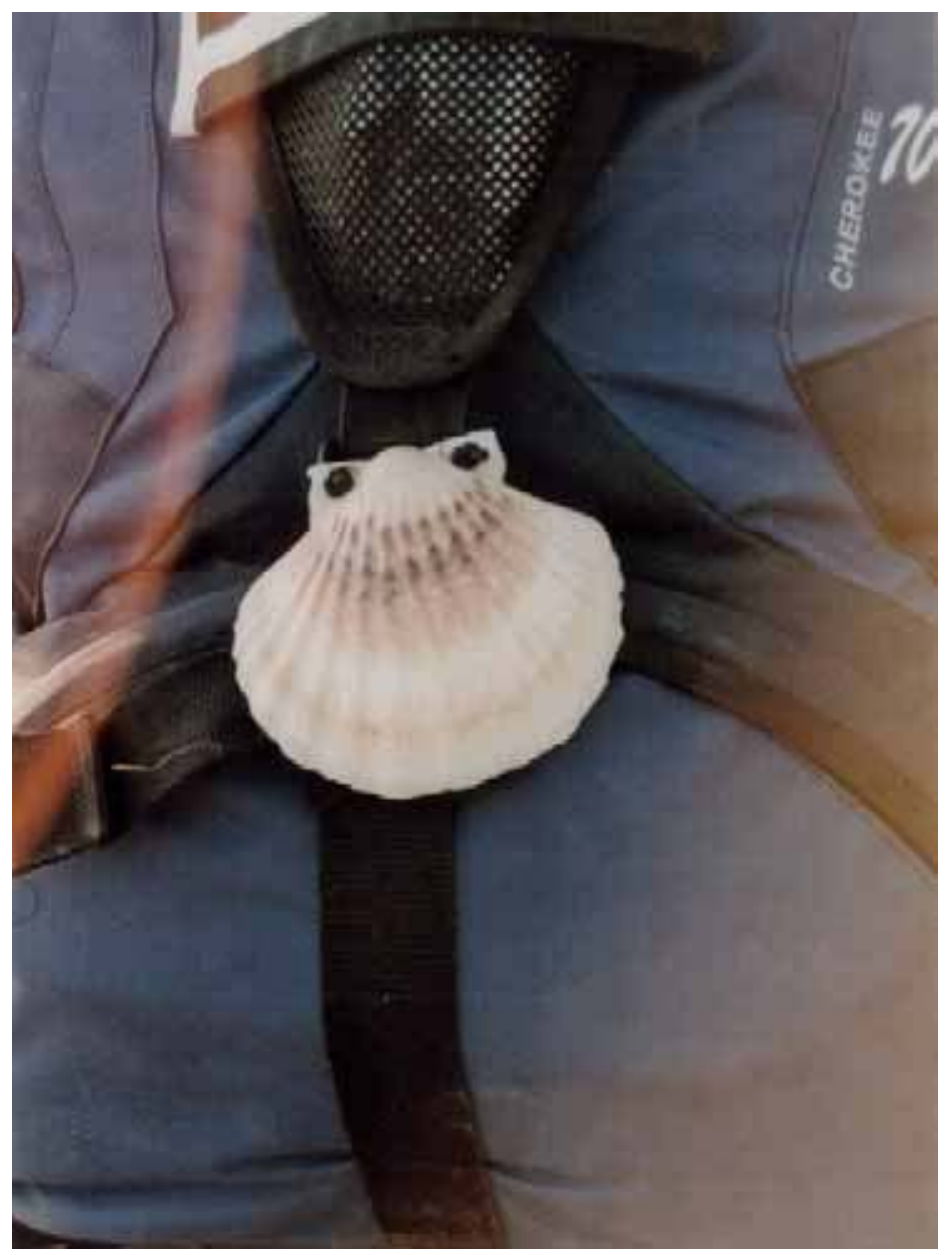

Coquille saint-jacques sur un sac à dos 
Il est intéressant de s'arrêter sur les objets contenus dans le sac: «J'ai cette bille. C'était une bille magique qu'une amie de la famille de mon amie m'a donnée. Le dernier jour, avant de venir ici, je suis allé les voir et ils m'ont dit : "Bonne chance pour ton voyage!" et Lisy a dit: "Prends cette bille dans ta poche, cela porte bonheur !" Et j'ai un journal où j'écris tous les jours. J'ai trois photos de mon enfant, trois ans et demi. J'ai un nickel, je l'ai toujours ; il vient d'Autriche. C'était le vieux shilling, avant l'euro. Je le lance et s'il y a le garçon sur le cheval, ça veut dire : "Vas !" et le numéro cinq veut dire "Stop !". Et à chaque fois que j'ai une question - mais ce n'est pas seulement pour la route, c'est la même chose chez moi -, c'est ma réponse mystique. Je n'ai pas mon téléphone portable... J'appelle la maison tous les trois ou quatre jours... (...) Mon enfant sait que je suis en train de marcher, je lui ai montré mon sac à dos et les choses dedans. Puis j’appelle ma mère, elle est angoissée : son fils est parti pour si longtemps ! » Dans ce bagage on remarque plusieurs objets « efficaces " pour le marcheur en chemin : le nickel pour choisir la route, la bille magique, les photos de son enfant, le carnet de voyage. La «bille magique », est dans ce cas l'objet spécial du pèlerin qui marque la sortie du milieu urbain, le rite du seuil et le passage au monde du chemin (Van Gennep, 1981 : 263-264) ; le nickel, déjà baptisé comme objet spécial pendant les détours urbains, lui apprendra la route telle une " réponse mystique »; les photos lui rendront la présence de son fils et son journal constituera une sorte de lieu de réflexion, un pont imaginaire entre l'ici du chemin - «Il y a quelque chose que je veux éloigner de moi en marchant » - et le là-bas de ses attentes "J'espère que quand je retournerai dans ma ville ou ma terre, j'aurai la réponse, j’aurai trouvé ».

Dans le bagage des voyageurs contemporains, figurent différentes catégories d'accessoires symboliques qui expriment une quête identitaire et un mode du croire : on y trouve des objets qui renvoient à la tradition catholique du pèlerinage (livret de prières ou de chansons du pèlerinage, le chapelet, la Bible) mais aussi des livres, des produits de soins non biomédicaux, des porte-bonheur, des objets chargés d'énergie (cristaux, pierres magiques) dont le poids est considéré comme une nécessité. Ces objets constituent des " médiums non verbaux » mais qui parlent à soi (Douglas, Isherwood, 1979 : 41) : pendant le temps du pèlerinage et tout au long de l'espace de la route ils représentent, traduisent et fixent matériellement les vœux identitaires des pèlerins.

Ce point est éclairci en tenant compte davantage du point du vue interne du pèlerin et des différentes logiques du voyage. Le témoignage suivant renvoie à l'éthique d'une existence minimaliste, recomposant l'idéal évangélique de la pauvreté qui alimente de nombreux rêves du départ. Dans ce cas, le Chemin de Compostelle est vécu comme un lieu de retour à la source du soi-essentiel, le "vrai moi-même ». Dans le langage des marcheurs affleure souvent, comme un exemplum, l'image de saint François allant à pied à Compostelle. La figure de ce saint, "chevalier errant " et «maître de ses rêves " (Frugoni, 1995: 10), sa 
sémantique de Madonna Povertà et son aspiration à une religion des mineurs, opérant à la frontière de la société ${ }^{6}$, convient aux pèlerins qui présentent souvent un « syndrome de saint François » contagieux. C'est le cas d'un Français employé d'Emmaüs International, âgé de quarante-huit ans, célibataire. Ce pèlerin parcourt la route sans argent; il va à Compostelle très lentement, en faisant des haltes de plusieurs jours dans les villages où il offre ses services comme cordonnier, son ancien métier, en échange de l'hospitalité : « J'ai l'essentiel avec moi, des choses pour réparer le cuir. C'est le côté que je voulais mettre en avant, je pars sans argent, je vis maintenant, je fais mon chemin tous les jours, je ne veux pas dépenser de l'argent sur le chemin, je veux que l'hospitalière me dise : “Tu donnes ce que tu veux". Si elle me parle comme ça, je l'aime, ça c'est le chemin. (...) C'est un chemin de spiritualité... Je ne peux pas dire... Il y a quelque chose... C'est catholique quand même, mais tu peux être protestant, tu peux être ce que tu veux. Je ne suis rien, je ne suis pas du tout... » Pourtant, Patrick se déplace avec le symbole de saint François, un tao: "C'est parce que quelqu'un me l'a donné, qui m'a dit qu'avec ça, ma vie serait plus belle et ma mort serait plus belle et avec ça, j'irai jusqu'au bout. » Cuir et tao font le bagage protestataire de ce cordonnier. Ces objets justes identifient sa recherche d'un lieu antisystème où il peut suspendre les normes d'une société qui, comme il le dit, "consomme trop ».

L'expérience essentielle de l'esprit qui habite nombre de marcheurs des classes moyennes et aisées - aussi bien chez les catholiques que chez ceux qui disent «n'appartenir à aucune religion »-, s'avère être, pour des pèlerins catholiques appartenant aux classes populaires, une pratique qui n'assume ni le caractère d'une esthétique du voyageur sans bagage ni celui d'une critique sociale. C'est de cela dont témoignent Pilar et Fátima, deux sœurs célibataires de Tenerife, " catholiques pratiquantes ", âgées respectivement de soixante-cinq et cinquante ans. Leur pratique de la route met en évidence les caractéristiques du pèlerinage populaire traditionnel ${ }^{7}$ : un voyage à pied vers le saint qui est à la fois une occasion extraordinaire pour voir le monde et une expérience d'émancipation rituelle. L'une des deux sœurs, Fátima, qui a déjà fait le chemin deux fois, décrit ainsi le pèlerinage : "Moi, je vais plus vite qu'elle, je m'arrête et je l'attends, elle ne peut pas faire beaucoup de kilomètres mais on se débrouille. Nous appelons tous les jours ma belle-sœur pour lui raconter et lui dire que cela se passe bien. »

6. Cf. aussi Victor W. Turner, The Ritual Process. Structure and Anti-structure, Chicago, Aldine Publishing Company, 1969, pp. 140-147, où l'auteur indique la figure du saint comme une image classique de l'idéal de vie itinérante, du refus de la structure sociale et du choix de la « liminalité » permanente.

7. Voir l'analyse d'Annabella Rossi, Le feste dei poveri, Palermo, Sellerio, 1986. Cette élève d'Ernesto de Martino décrit le pèlerinage catholique populaire, en se référant, en particulier, au sud de l'Italie, comme un voyage qui permet de régénérer et de " rassurer le quotidien » par le fait qu'il offre des rôles extraordinaires et qu'il répond à des exigences individuelles et sociales peu canalisables autrement. 
Parmi mes informateurs, ces deux sœurs ont le record du sac le plus léger : « Le sac est petit, il ne pèse pas plus de six cents grammes. Il y a le nécessaire pour la toilette, des vêtements d'été s'il fait chaud et des sous-vêtements. Le guide, oui, c'est moi qui le porte, il est nécessaire, oui. Et puis je l'ai rempli de chansons et de refrains du Chemin. » Dans leur bagage, des objets nécessaires au rite de passage matériel, se mélangent au livret de prières et de chansons du pèlerinage considérés comme des objets efficaces pour le passage symbolique.

L'expérience de l'essentiel et du vécu avec le peu d'objets indispensables à la vie pèlerine est décrite comme transformative : "J'ai eu une expérience frappante, c'est quelque chose qu'on ne peut pas expliquer et, moi, j'ai changé totalement de vie. Tu te rends compte que pour vivre il ne faut pas grand chose. On se rend compte que pour vivre beaucoup de choses ne sont pas nécessaires. Avec l'essentiel tu en as plus qu'assez. " Cette dernière phrase, "Avec l'essentiel tu en as plus qu'assez " ( Con lo esencial vas de sobra») constitue un paradoxe significatif. Dans ce témoignage, avoir peu devient une stratégie de liberté - là où pour le cordonnier il s'agissait d'un choix de protestation sociale - et un moteur opérant vers une recréation du soi qui sera perçue lors de la réintégration à la vie urbaine; l'expérience du " poids essentiel » devient centrale dans la réussite du rite de passage que le pèlerinage représente.

L'analyse d'un autre bagage permet d'approfondir la réflexion sur les croyances des « jacquaires " contemporains. Il s'agit d'un aspirant écrivain danois, âgé de trente-trois ans, ayant reçu une éducation protestante mais qui se place actuellement dans une position spirituelle qu'il définit comme relevant d'une "religion non organisée ». Ce jeune homme, qui se qualifie comme un walker plutôt que comme " pèlerin » et qui marche " léger ", est très attentif à ses objets essentiels. Il a deux tenues, une pour le jour et une pour le soir, mais il a fait le choix de ne prendre ni guide, ni sac de couchage (ce dernier, qui pèse environ un kilo et demi, est considéré en général comme indispensable). Par contre, il ne renonce pas à son journal, à son stylo fétiche et au livre d'Ernest Hemingway A Farewell to Arms, son «introduction à l'Espagne ". Dans cet équipement, il n'y a aucun objet faisant référence au pèlerinage de Saint-Jacques-de-Compostelle ; le bagage est semblable à celui que ce marcheur pourrait amener avec lui dans d'autres voyages, comme il l'affirme lui-même. Le contenu de son sac suggère une individualisation de la route qui ne correspond ni au label religieux "pèlerinage » ni à celui touristique-culturel du "Chemin de Compostelle». De cette déclinaison de la condition de l'essentiel émerge l'élaboration personnelle d'une mythologie de la route et l'attitude qu'on remarque ici est plutôt celle d'un walking planétaire qui correspond à un système croyant sur mesure : le chemin du good fellow Jago - ainsi appelle-t-il le chemin de saint Jacques - s'avère être configuré comme une " piste utopique » bricolée selon la foi en un panthéon privé. Mariana, une jeune étudiante en architecture, Brésilienne de Rio de Janeiro, en échange universitaire à Barcelone, part à la fin de l'année scolaire vers Compostelle. Élevée 
dans le catholicisme mais non pratiquante, elle affirme faire " un chemin de méditation mystique " et s'intéresser à l'histoire et à l'architecture romane en Espagne. Dans son sac, il y a un objet caché, qui fait rêver lorsqu'elle en parle aux autres marcheurs : une robe blanche, gardée intacte, à utiliser uniquement le jour de son arrivée à Compostelle " pour entrer propre dans la cathédrale ". La pureté gardée dans son équipement et portée sur soi tout au long du parcours, va sortir du sac à la fin du voyage. Selon la quête de la jeune Brésilienne, toutefois, la valeur transformatrice du vêtement blanc fonctionne déjà en route, lorsqu'elle porte le poids de l'objet. Le résultat de cette interprétation de la route est le «Chemin » enchanté d'une "pèlerine non catholique ».

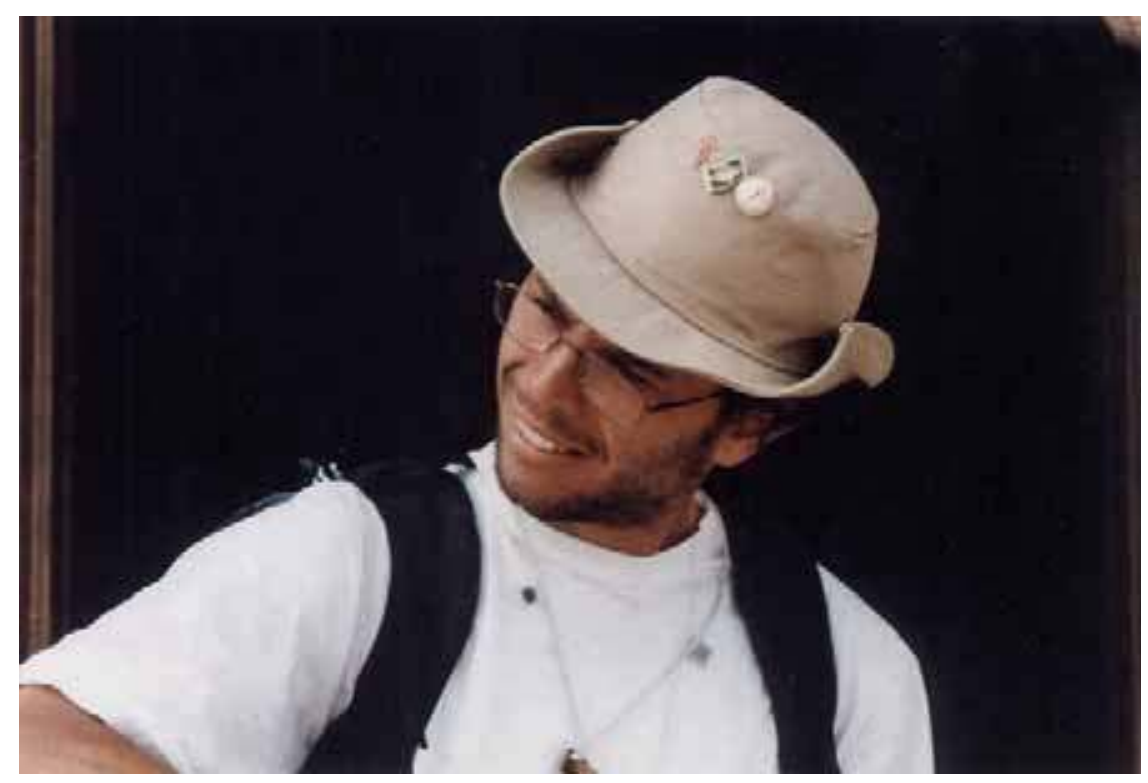

Pèlerin brésilien sur le chemin de Saint-Jacques de Compostelle

\section{Le bricolage du stock de la mémoire et la métaphore du Chemin}

Les bagages de ces quelques marcheurs montrent la scène de Compostelle comme un laboratoire de métissage spirituel marqué, d'une part, par la crise de la régulation institutionnelle du pèlerinage et, d'autre part, par le réaménagement de l'offre catholique. En marchant, on peut s'inscrire dans la lignée croyante catholique ou alors puiser dans le stock de la mémoire du pèlerinage, choisir parmi les multiples traditions disponibles et bricoler sa propre voie; on peut ainsi mélanger la foi chrétienne à l'ésotérisme celtique ou à la quête mystique sous l'orme des chevaliers de Charlemagne ou à l'engagement New Age dans une expérience de chemin magico-initiatique de développement personnel. Cette tendance à la réinvention du pèlerinage catholique est d'ailleurs explicitée par les 
pèlerins lorsqu'on leur demande s'ils s'identifient avec la définition "pèlerin/e " et s'ils identifient leur acte de marcher avec le mot " pèlerinage ». Plusieurs informateurs, interrogés à ce sujet, répondent qu'ils préfèrent se décrire comme un/une simple marcheur/euse, un/une caminante, un/une walker, comme l'indiquait déjà le témoignage du jeune Danois cité plus haut.

La double dénomination de la scène de Compostelle, comme "pèlerinage » et comme "camino » est fondamentale pour comprendre le fonctionnement du phénomène observé : cette oscillation sémantique explicite les écarts de sens existant entre la logique du pèlerinage catholique et celle des chemins individuels. Cette disjonction indique aussi que la notion de "pèlerinage ", référée à la scène de Compostelle, doit être comprise non seulement dans un sens anamnestique, comme un acte qui fait mémoire de la tradition catholique et en relation à son histoire de peregrinatio maior de la chrétienté, comme Rome et Jérusalem, mais aussi dans un sens métaphorique, comme synonyme de chemin, de voie, de parcours de transition identitaire. Une jeune Espagnole de Cadix, catholique pratiquante, souligne le pouvoir métaphorique de l'expérience du Chemin : « Je veux arriver à Santiago. Je veux absolument arriver à Santiago et embrasser le saint, je dois le faire parce que, si je le fais, si je réussis, je pourrai réussir d'autres choses dans ma vie. Le Chemin m'a formée de telle façon que, maintenant, je suis capable d'attendre, d'endurer beaucoup. En fait, j'assimile une chose à l'autre, ainsi je dis: "Si je suis capable d'arriver là-bas, alors je crois que dans la vie... oui, si je le fais, je suis capable de supporter et d'arriver où je veux. »

La même métaphore du Chemin comme parcours symbolique de l'aller de l'avant dans la vie est également présente dans le discours des marcheurs qui ne se définissent pas comme catholiques. Dans la construction sociale de cette métaphore, la logique de l'épreuve quotidienne qu'implique le long pèlerinage à pied joue un rôle central : la structure du voyage en étapes constitue une métaphore de l'aller de l'avant et du faire sa propre voie qui fournit un canevas excellent pour la fabrication d'un soi nouveau. ${ }^{8}$ Dans le corps-à-corps avec la route, le sujet se forge lui-même par une entreprise inhabituelle demandant une résistance, une volonté de parvenir au but et une sorte de qualification charismatique. Roberto, entrepreneur de Madrid, âgé d'une quarantaine d'années, se définissant comme une "personne religieuse mais pas catholique », explicite cette dynamique de construction de soi en héros : " Les gens t'arrêtent quand tu arrives à Santiago et ils te demandent avec admiration d'où tu viens, les touristes te prennent en photos, tu te sens le héros du jour, le jour où tu arrives et que tu vas à la messe du pèlerin, c'est ta messe de pèlerin. » Cet entrepreneur non catholique

8. La structure du voyage en étapes fournit par ailleurs une source d'inspiration aux auteurs contemporains intéressés par la thématique de la métamorphose personnelle. Le best-seller de Paulo Coelho, cité plus haut, ainsi que celui de genre New Age, de Shirley Mac Laine, Mon chemin vers Compostelle. Un voyage de l'esprit, Paris, Plon, 2002, l'indiquent clairement. 
" impliqué paradoxalement " ${ }^{9}$ dans les rites de l'arrivée, qui se couronne lui-même comme pèlerin dans le lieu de la cathédrale, décrit le pèlerinage comme un « autre monde » dont les dynamiques d'accès et de sortie sont déterminées uniquement par l'acte de l'aller : «Mais, quand je suis arrivé à Santiago, ce moment a été très éphémère, ça a duré quelques heures. Après l'arrivée et l'entrée sur la place de l'Obradoiro, après la messe du pèlerin, tu ressens déjà comme si la ville te repoussait pour laisser entrer de nouveaux pèlerins. Tu reviens un peu à l'introspection, tu commences à te souvenir d'endroits plus beaux ou plus naturels que Santiago. Santiago c'est très touristique et tu commences à te rappeler, enfin, des villages par lesquels tu es passé en Castille, du gîte où ils ont dû t'inviter à dîner parce que tu n'avais rien à manger, du gîte où l'hospitaliero a soigné ton ampoule en y mettant de l'eau et du sel. Alors, tu essayes de rattraper tout le Chemin dans ta tête et cela tu le fais pendant longtemps après avoir terminé le Chemin, c'est là où est le vrai enseignement du Chemin. "

L'accent sur la performance extraordinaire du pèlerin et sur l'altérité du «monde du Chemin » est davantage explicité par la description du moment du retour chez soi, en ville : "Ce qui se passe après le Chemin c'est que tu ressens une très grande confiance en toi-même. Je crois que tout le monde, quand il termine le Chemin, revient chez lui, en ville, comme si il n'appartenait pas à la ville, comme si les gens autour de lui appartenaient à un autre monde, tu reviens chez toi et tu dis: "Moi je suis différent de ces gens, je suis capable de faire sept cents kilomètres à pied ". Comme le suggère ce témoignage, el Camino se présente comme un voyage dans le temps, déterminé par l'espace. Dès le départ, ce voyage sort celui qui l'entreprend de l'espace et du temps ordinaires. En marche, le temps s'écoule de manière extraordinaire et les heures et les jours passent différemment : on marche tout le temps; on ne regarde la montre que pour calculer l'heure d'ouverture des gîtes, de la fermeture des boutiques alimentaires ou d'une éventuelle messe; on rentre dans un univers à forte temporalité présente qui permet de « congeler » le monde quotidien. Dans ce contexte, où ce n'est pas la condition professionnelle qui est productrice d'identité, habillé en pèlerin, en portant tous les jours les mêmes vêtements et sans les accessoires du quotidien, on peut raconter aux autres son identité quotidienne et son rôle social de jure ou se taire à ce sujet, en se choisissant un rôle présent de facto, déterminé par un unique accent majeur: "Je suis le chemin que j'ai parcouru ».

Cette formule signifie que, sur la scène de Compostelle, on accorde une valeur absolue à la réalisation de l'individu in itinere et, pour cette raison, cette scène est un univers d'interaction informelle, à dimension utopique, où l'on a l'impression d'être " tous égaux ». Dans ce monde, surtout lors des premiers jours d'une

9. Cette expression est utilisée pour rendre compte de l' « engagement partiel » et de l' « identification latérale » qui caractérise l'adhésion aux rites de l'arrivée de ce pèlerin non catholique. Voir Albert Piette, "Implication paradoxale, mode mineur et religiosités séculières ", Archives de Sciences Sociales des Religions, 81, 1993, pp. 63-78. 
rencontre, on existe pour l'autre marcheur uniquement par un prénom, une nationalité et le nombre de kilomètres que l'on a parcouru : on est le Tchèque qui vient à pied de Prague, la Québécoise qui marche depuis Le Puy-en-Velay, le Breton qui arrive du Finis Terrae de Quimper, la Japonaise qui est arrivée sur le Chemin en passant par Lourdes. En considérant cela, on comprend que le paramètre de la validité sociale mis en scène sur la route est différent de celui de la vie quotidienne ; en chemin, l'âge et la profession constituent des critères d'identification mineurs, qui émergent quand une compagnie de marcheurs se consolide dans le temps, mais qui, cependant, dans la société de Compostelle, ne constituent pas les termes d'affirmation d'un soi réalisé. Ce qui compte c'est d'où l'on vient à pied, depuis combien de temps on marche et combien de pays et de villages on a traversés : l'identité pèlerine se réalise en allant. C'est avec l'agencement individuel de la puissante métaphore du Chemin et avec sa disjonction du discours croyant traditionnel que doit compter actuellement le pèlerinage catholique.

\section{La mise en scène d'un drame de la présence}

Les configurations existentielles qui déterminent la décision de faire le pèlerinage permettent d'éclairer davantage les logiques de départ vers Compostelle. Le « chemin » ou " pèlerinage ", selon le penchant spirituel du marcheur, est une expérience vers laquelle on se tourne souvent à la suite d'une crise identitaire, d'un deuil, d'un divorce, d'une phase d'incertitude ou d'une crise professionnelle, la retraite ou le chômage ou, plus généralement, pour marquer un arrêt par rapport à un rythme de vie quotidien perçu comme trop accéléré. L'habit du pèlerin semble répondre à un besoin global de régénération identitaire : en faisant son chemin, grâce à l'épreuve de la marche, on se fait « entrepreneur de soi-même » (Ehrenberg, 1991), on se constitue comme performant, et, ainsi, on rêve d'endiguer symboliquement la précarité qui fragilise le quotidien dont on s'est séparé.

Une pèlerine française de Nîmes, catholique pratiquante, âgée d'une cinquantaine d'années, veuve, retraitée de son travail de secrétaire générale d'une inspection académique dans l'administration de l'éducation nationale, explique : "J'ai une amie qui a fait le chemin il y a cinq ans et qui est revenue enchantée de ce voyage mais elle l'avait fait dans un but particulier. Elle venait de perdre, deux ou trois ans avant, sa fille qui s'était suicidée et elle n'arrivait pas à reprendre le dessus. Donc elle a fait ce chemin en parlant de sa fille en permanence et en parlant à sa fille et c'est une femme qui est revenue complètement transformée par rapport à ça et maintenant elle vit normalement. Elle a surtout vu à travers ce voyage que sa fille est vivante là où elle est et que sa fille lui a dit : "Profite de la vie que tu as sur terre." Donc, chemin faisant, j’en ai parlé avec ma bellesœur ; moi je voulais le faire il y a trois ans quand j'ai pris ma retraite... Ma belle-sœur m'a dit : “Attends-moi.” Entre temps, je n'avais plus envie d'y aller 
et, à partir du moment où elle était libre, elle m'a dit: “Bon, on le fait !” Et moi, toute l'année, j'étais mal fichue et j'avais mal au genou. J'étais embêtée et je l'ai dit au Seigneur, parce que je suis très catholique... Personne de ma famille ne pouvait venir avec moi. Mon mari est décédé il y a quatre ans quand je travaillais... Donc, une semaine avant de partir, je n'étais pas sûre. Il y a trois ans, quand j'ai voulu le faire, je voulais extirper un certain nombre de choses, la mort de ma mère, la mort de mon mari, la retraite, le divorce de mon fils, et il fallait une thérapie et mon but c'était d'aller à Santiago pour faire le tri dans tout ça et placer ces choses dans l'ordre où il fallait qu'elles soient... J'étais perturbée par ces quatre évènements et il fallait qu'ils ne me perturbent plus, sans pour autant les nier, il fallait qu'ils soient à leur bonne place. C'est une thérapie pour moi, la marche. C'était pour replacer les choses là où il fallait les placer, ne pas leur donner plus d'importance qu'elles doivent avoir, tout en les respectant, que moi je puisse continuer à vivre normalement. »

David, jeune Espagnol élevé catholique mais non pratiquant, âgé de vingtcinq ans, employé dans un café de son village, décide de ne pas s'inscrire à l'Université et d'entreprendre le chemin pour essayer de trouver une direction dans la vie: "Si tu n'es pas croyant, le Chemin sert pour ouvrir les yeux sur la vie. C'est-à-dire que le Chemin est comme le box d'une piste de course : tu mènes une vie sans direction, tu te mets sur le Chemin et comme si tu étais une voiture de course au pit stop, ils te remettent au point et alors tu prends ta direction et tu continues, continues, continues. »

L'image paradoxale d'une vitesse extrême utilisée pour décrire la lenteur du pèlerinage à pied, souligne l'expérience du chemin comme un temps de réorganisation de la vie, stratégie à emprunter lors d'une fragilisation identitaire et d'un moment d'incertitude dans la « course de la vie ». Ce sentiment apparaît souvent dans la génération des jeunes adultes au seuil de la vie professionnelle et aussi chez les pèlerins les plus âgés qui envisagent la sortie du monde du travail. Les adultes, par contre, fréquemment impliqués dans un vécu professionnel très actif, décrivent le chemin comme une pause régénératrice.

Une psychologue française, séparée, mère de deux enfants, qui atteste d'un bricolage spirituel à tendance bouddhiste sur fond de culture catholique et qui parcourt le chemin de Compostelle en séries d'étapes annuelles, décrit le pèlerinage comme un lieu de retraite : "La raison de mon pèlerinage c'est de pouvoir, pendant un certain temps, changer mon rythme de vie et de vivre au rythme de la marche. J'ai un rythme de vie dans le quotidien qui est très accéléré, qui est très astreignant, et je suis toujours dans une relation d'être avec les autres, dans une dynamique permanente avec les autres, et le fait de faire le chemin de Compostelle c'est pour moi une façon de me remettre un petit peu face à moi-même et de me mettre sur un rythme très lent, et de vivre sur un rythme lent, et c'est quelque chose qui, pour moi, a été très enrichissant l'année dernière... et c'est une forme de retraite, je crois, on marche parce que c'est une forme de retraite. » Les motivations des retours annuels sur le chemin sont davantage explicitées comme une 
pratique de méditation en accord avec la pratique du bouddhisme : "C'est le fait de pouvoir se détacher de toute cette agitation au niveau mental, au niveau du vécu que l'on a dans la civilisation actuelle, se retrouver en contact avec le divin pour une intériorisation en plus, et je trouve que dans les édifices religieux que l'on rencontre sur le chemin de Compostelle, on rencontre vraiment cette connexion avec le divin. Dans la plupart des édifices religieux, il y a une sérénité et, à travers l'architecture, je me trouve dans la même connexion que celle que je retrouve dans la pratique bouddhiste. Psychologiquement, ça me clarifie l'esprit, ça me repose mon mental et ça me donne le même sentiment que quand je fais une retraite spirituelle. » Si l'on retient ces témoignages, le chemin, selon le dire des pèlerins, lieu " merveilleux » et " magique », apparaît aussi comme le baromètre social d'une incertitude sociale et d'une inquiétude identitaire diffuse. Les sujets qui se construisent comme pèlerins mettent en place, par l'activité de l'aller - cet acte de progression concrète et en même temps symbolique - un "drame de la présence " ${ }^{10} \mathrm{ou}$, selon une expression très caractéristique des pèlerins euxmêmes, un drame de la quête, et aussi la possibilité de sa résolution en surmontant l'épreuve du chemin.

Face au sentiment individuel d'une fragilisation de son monde, le mouvement pas à pas du pèlerin devient une stratégie thérapeutique, qui agit de façon "homéopathique » sur la mobilité extrême de la société contemporaine : en se mettant en scène comme pèlerin, on répond par un mouvement "actif » et " orienté " à un mouvement du quotidien où le temps et l'espace sont souvent perçus comme des conditions subies plutôt qu'agies.

Dans ce contexte, cette pratique de pèlerinage, qui consiste en une activité volontaire, modulable, dont on peut faire usage, si l'on veut, comme d'une tradition religieuse collective mais aussi comme d'une pratique spirituelle individuelle, offre la possibilité de concrétiser un «processus de subjectivation de l'utopie » (Hervieu-Léger, 2003 : 148). Le pèlerinage/Camino contemporain est construit sur mesure comme un espace utopique - c'est-à-dire un espace qui accueille « un appel au passé contre le présent en vue d'un avenir » (Séguy, 1999: 119) - qui permet une implication religieuse paradoxale et un jeu de marge pour ce qui est de l'engagement croyant catholique.

\section{Elena ZAPPONI \\ Rome, Université de la Sapienza - Paris, EPHE-GSRL elenazap@yahoo.com}

\footnotetext{
10. Selon Ernesto de Martino, ce drame consiste dans le déroulement d'une action positive face à une " crise de la présence » du sujet déterminé par un quotidien qui, n'étant plus maîtrisé, implique le risque du non-être : de Martino, Il mondo magico, [1948] 1973, Torino, Boringhieri, pp. 94-95 ssq. Ce concept de drame de la présence originairement élaboré en relation au magisme est repris dans l'analyse du phénomène du tarantisme dans E. de Martino, La terre du remords, (La terra del rimorso, 1961), Paris, Institut Synthélabo, 1999.
} 


\section{Bibliographie}

Bertrand Jean-René, Muller Colette, 1999, Religions et territoire, Paris, L'Harmattan. Coelho Paulo, 1987, Le pèlerin de Compostelle (O Diario de um mago), Paris, Éditions Anne Carrière.

De Martino Ernesto, Il mondo magico, 1973 (1948), Torino, Boringhieri.

-, 1999, La terre du remords, (La terra del rimorso, 1961), Paris, Institut Synthélabo.

DOuglas Mary, IsHeRwood Baron, 1979, The World of Goods, New York, Routledge.

Dupront Alphonse, 1987, Du sacré. Croisades et pèlerinages. Images et langages, Paris, Gallimard.

Ehrenberg Alain, 1991, Le culte de la performance, Paris, Calman-Lévy.

Frugoni Chiara, 1995, Vita di un uomo : Francesco d'Assisi, Torino, Einaudi.

Hervieu-LÉger Danièle, 1999, Le pèlerin et le converti. La religion en mouvement, Paris, Flammarion.

-, 2003 Catholicisme, fin d'un monde, Paris, Bayard.

LÉVI-STrauss Claude, 1974, Anthropologie structurale, Paris, Plon.

PIETTE Albert, 1993, "Implication paradoxale, mode mineur et religiosités séculières ", Archives de Sciences Sociales des Religions, 81, pp. 63-78.

Rossi Annabella, 1986, Le feste dei poveri, Palermo, Sellerio.

SÉGUY Jean, 1999, "Une sociologie des sociétés imaginées, monachisme et utopie ", in Conflit et Utopie, ou réformer l'Église. Parcours wébérien en douze essais, Paris, Éditions du Cerf, pp. 111-159.

Turner W. Victor, Turner Edith, 1978, Image and Pilgrimage in Christian Culture. Anthropological Perspectives, Oxford, Blackwell.

Internet : www.archicompostela.org

\section{Résumé}

Le pèlerinage de Compostelle est aujourd'hui une métaphore de l'aller de l'avant et de la vie comme voyage, attirant un public international et intergénérationnel bien plus vaste que les seuls croyants catholiques. La scène de Compostelle offre un espace ouvert tant à l'expérience religieuse inscrite dans la tradition chrétienne-catholique qu'à une quête identitaire, qui exprime une individualisation $d u$ Chemin et une prise de distance vis-à-vis de l'Église sur le pèlerinage institutionnel. À partir des données de terrain, une perspective précise est proposée pour comprendre les recompositions $d u$ croire qui caractérisent la route de Compostelle : l'analyse du bagage des pèlerins, et en particulier de leurs objets, permettra de saisir les croyances des sujets qui les portent.

Mots-clés : Saint-Jacques-de-Compostelle, pèlerinage, recomposition du croire, rite de passage, individualisme religieux.

\section{Abstract}

The pilgrimage to Santiago de Compostela has become a metaphor for going forth and life as a journey and is no longer only travelled by Catholics but attracts a much larger international and intergenerational public. The surroundings are conducive to both the religious experience within the Catholic Christian tradition and to a self 
identity quest, personalising the pilgrimage's tradition and distancing it from the Church's point of view. The author proposes a specific methodological perspective to approach the believing reconstruction on the pilgrim's road: the observation of pilgrims' backpack and in particular of their objects, allows to understanding the believing of their owners.

Key words: Saint James of Compostela, pilgrimage, reconstruction of believing, rite of passage, personalization of believing.

\section{Resumen}

La peregrinación hacia Santiago de Compostela se ha convertido en una metáfora del seguir adelante y del avanzar en la vida como en un viaje; el público que lo practica no está compuesto sólo por católicos sino por un abanico de actores internacionales e intergeneracionales. Esta escena peregrina ofrece un espacio abierto tanto a la experiencia religiosa que se inscribe en la tradición católico-cristiana como a una búsqueda identitaria que expresa una individualización del camino y una toma de distancia en relación a la noción de peregrinación propuesta por la Iglesia. El autor propone un enfoque metodológico específico para acercarse a las recomposiciones del creer que caracterizan esta ruta peregrina: el análisis del equipaje de los peregrinos y, en particular, de los objetos que llevan consigo durante el camino. A partir de estos objetos, se analizan las creencias de los peregrinos.

Palabras clave: Santiago de Compostela, peregrinación, recomposición del creer, rito de pasaje, individualización del creer. 